\title{
Influência da Fadiga Ocupacional na Capacidade para o Trabalho de Professores Universitários
}

\author{
INFLUENCE OF OCCUPATIONAL FATIGUE IN THE WORK ABILITY OF PROFESSORS
}

Thayla Amorim Santino', Alecsandra Ferreira Tomaz², Neide Maria Gomes de Lucena ${ }^{3}$

1. Mestranda em Fisioterapia do Centro de Ciências da Saúde, Universidade Federal do Rio Grande do Norte (UFRN), Natal, RN, Brasil. Fisioterapeuta graduada pela Universidade Estadual da Paraiba (UEPB), Campina Grande, PB, Brasil.

2. Doutoranda em Engenharia de Processos, Universidade Federal de Campina Grande (UFCG). Doutorado em Medicina do Esporte, Universidad Catolica Nuestra Senora de La Asuncion, Paraguai. Professora do Departamento de Fisioterapia, Universidade Estadual da Paraíba (UEPB), Campina Grande, PB, Brasil.

3. Pós-Doutorado na Universidade de Granada, Espanha. Doutorado em Educação Fisica, Universidad de Granada, Espanha. Professora titular do Departamento de Fisioterapia, Universidade Federal da Paraiba (UFPB), João Pessoa, PB, Brasil.

\section{RESUMO}

0 objetivo deste estudo foi verificar a influência da fadiga ocupacional na capacidade para o trabalho de professores do Centro de Ciências Biológicas e da Saúde de uma Instituição de Ensino Superior (IES) pública do Brasil. Trata-se de um estudo transversal descritivo, de abordagem quantitativa, realizado com 89 professores universitários da cidade de Campina Grande/PB. Foi utilizado um Questionário de Características Sociodemográficas e Laborais, o Questionário de Índice de Capacidade para o Trabalho (ICT) e o Questionário de Fadiga de Yoshitake (QFY). Os resultados evidenciam que a maior parte da amostra $(70,9 \%)$ apresentava boa capacidade para o trabalho e um moderado nível referido de fadiga, sendo esta de ordem mental. De acordo com a análise inferencial foi visto que à medida que aumenta a presença da fadiga, diminui-se a capacidade para o trabalho $(r=-0,48 ; p<0,0001)$. Além disso, foi encontrada associação entre o sexo $(p=0,004)$ e a presença de problemas para dormir $(p=0,000)$ com a ocorrência de fadiga ocupacional.

(Amorim T, Ferreira A, Gomes de Lucena N, 2017. Influência da Fadiga Ocupacional na Capacidade para o Trabalho de Professores Universitários. Cienc Trab. May-Ago; 19 [59]: 86-90).

Palavras-chave: DOCENTES, SAÚDE DO TRABALHADOR, FADIGA.

\section{ABSTRACT}

The aim of this study was to verify the influence of occupational fatigue in work ability of professors of the Centro de Ciências Biológicas e da Saúde from a Higher Education Public Institution (HEPI) of Brazil. This is a descriptive cross-sectional study with a quantitative approach, carried out with 89 university teachers of Campina Grande/PB. It was used a Sociodemographic Characteristics and Employment questionnaire, Work Ability Index Questionnarie and Fatigue Questionnaire. Results shows that the majority of the sample $(70,9 \%)$ had good work ability and a moderate level of fatigue, which is mental. According to the inferencial analysis, as the presence of fatigue increases, the work ability decreases $(r=-0,48$; $\mathrm{p}<0,0001)$. In addition, it was found association between sex $(\mathrm{p}=$ $0.004)$ and the presence of sleep problems $(p=0.000)$ with the occurrence of occupational fatigue.

Key words: PROFESSORS, OCCUPATIONAL HEALTH, FATIGUE.

\section{INTRODUÇÃO}

A partir da Revolução Industrial evidenciou-se a existência de uma relação direta entre a atividade laboral e o processo saúde-doença. ${ }^{1}$ Com o avanço tecnológico, modificações na política educacional e

Correspondência / Correspondence:

Alecsandra Ferreira Tomaz

Centro de Ciências Biológicas e da Saúde, Departamento de Fisioterapia, Universidade Estadual da Paraiba

Av. das Baraúnas, 351, Campus Universitário, Bodocongó

Tel.: +55 (83) 3315-3346

e-mail: alecsandratomaz@hotmail.com

Recibido: 30 de Enero 2017 / Aceptado: 27 de Junio de2017 na organização do trabalho, a atividade docente também sofreu mudanças. ${ }^{2}$ Desde o século XIX, o reconhecimento e a relevância social do professor se elevaram, porém, é uma profissão que requer extrema dedicação e, por vezes, está associada ao desgaste físico e mental. ${ }^{3,4}$ Nesse sentido, o trabalho que deveria ser uma forma de realização e reconhecimento, pode tornar-se um fardo e afetar a capacidade para o trabalho. ${ }^{5}$

As cargas psicológicas aumentaram, bem como as diversas exigências laborais, relacionadas à docência, competitividade e reconhecimento necessário no meio acadêmico. Atualmente, as atividades dos docentes ultrapassam as barreiras da sala de aula, envolvendo também a gestão e o planejamento de ensino, pesquisa e extensão. 0 profissional está sujeito a elevadas cargas de trabalho, diante disso, ocorre uma maior dificuldade na organização do trabalho. Longas jornadas resultam em menos tempo disponivel para o convívio familiar, consequentemente encontra-se mais sujeito ao desgaste físico e psicológico. ${ }^{1,2,4,6}$ 
Considerando os desgastes das capacidades físicas e mentais, verifica-se a ocorrência de fadiga ocupacional, uma vez que a docência predispõe o trabalhador a fatores que estão interligados. Sendo assim, a fadiga é exposta como um dos agravos decorrentes de atividades laborais. A mesma é caracterizada como um fenômeno multidimensional que compreende aspectos fisiológicos e psicológicos e está relacionada à sensação de cansaço, falta de energia e exaustão, que resulta na diminuição da capacidade de realizar atividades cotidianas. Além disso, a fadiga altera o estado de alerta e vigília, assim como a motivação. ${ }^{7,8}$

0 trabalho docente é caracterizado por envolver modelos de produção e prestação de serviços que exigem uma adequação em um formato acelerado e intensificado, indicando um aumento da produtividade através do aumento do ritmo, da carga, da responsabilidade de trabalho, e da redução das pausas para descanso. ${ }^{5}$ Observou-se que o exercício da docência sofre influência de diversas condições, sobretudo, as condições do ambiente, mobiliário, estrutura física, carga horária, tipo de atividade, relacionamento interpessoal, características relacionadas ao prazer em realizar a atividade laboral, sobrecarga de trabalho, entre outras. ${ }^{1}$ 0 exercício da docência relacionado com a precarização das condições laborais, está levando ao crescente aumento de transtornos de saúde em docentes, refletindo diretamente no trabalho. Estudos evidenciam que a capacidade para o trabalho está interligada aos aspectos sociodemográficos, estilo de vida, saúde (capacidade funcional, mental e física) e às condições de trabalho e da função desempenhada. Observa-se que a satisfação no trabalho, tempo de serviço, cargo, turno, carga horária, autonomia e controle são fatores que irão intervir, bem como o estado de saúde, entre outros., ${ }^{4,9}$

A partir da década de 1990, ocorreu um aumento nos estudos envolvendo tais aspectos, relacionando a repercussão do trabalho sobre a saúde física e mental. Tal aumento foi justificado mediante o crescente número de docentes que sofrem com a fadiga física e/ ou exaustão mental, sendo assim, relacionado com a importância da realização das produções científicas apresentando abordagem direta sobre as condições de saúde do professor universitário. ${ }^{1,10}$ Estudos dos níveis de fadiga e capacidade para o trabalho proporcionam a obtenção de uma medida relevante em saúde através da identificação precoce da ocorrência de sobrecargas e a compreensão dos fatores envolvidos no desenvolvimento. ${ }^{8}$

Diante do exposto, o objetivo desta pesquisa é verificar a influência da fadiga ocupacional na capacidade para o trabalho de professores do Centro de Ciências Biológicas de uma IES pública da cidade de Campina Grande/PB.

\section{MATERIAIS E MÉTODOS}

Trata-se de um estudo transversal, de caráter descritivo, de abordagem quantitativa, retirado de um projeto maior intitulado "Influência da Capacidade para o Trabalho na Condição de Saúde dos Professores de uma IES Pública”.

\section{Participantes do estudo}

A população que constitui este objeto de estudo foi composta por professores universitários do Centro de Ciências Biológicas e da Saúde da Universidade Estadual da Paraíba (UEPB), localizado na cidade de Campina Grande/PB, Brasil.

Para fins de cálculo amostral, a população considerada foi de 242 professores efetivos dos sete cursos do Centro de Ciências Biológicas e da Saúde da referida Universidade. Utilizando uma margem de erro de 5\% e fórmula para cálculo de amostras finitas, obteve-se o número de 151 professores. Utilizou-se amostragem por conveniência, mediante disponibilidade dos sujeitos de participarem da pesquisa.

Foram considerados como critérios para inclusão: professores do Centro de Ciências Biológicas e da Saúde da UEPB que estivessem lecionando no período da coleta de dados. E como critérios de exclusão: professores substitutos ou convidados da referida Instituição.

\section{Procedimentos para coleta dos dados e instrumentos de pesquisa}

Os instrumentos para coleta de dados foram previamente testados em um estudo piloto com 25 docentes da referida Universidade. Após os ajustes necessários, a coleta de dados foi iniciada e realizada através do método de entrevistas com pesquisadores treinados, sendo estes alunos do Departamento de Fisioterapia da referida Instituição de Ensino. Ao final da coleta, a amostra foi composta por 89 docentes.

Como instrumentos para coleta de dados foram utilizados: Questionário de Características Sociodemográficas e Laborais, contendo questões acerca das características do trabalhador, como sexo, idade, estado civil, escolaridade e questões sobre as atividades laborais; o Índice de Capacidade para o Trabalho (ICT) e o Questionário de Fadiga de Yoshitake (QFY).

0 ICT é um instrumento que permite obter uma medida preditiva sobre o perfil geral da capacidade para o trabalho, capacidade funcional e fatores que os afetam. Composto por sete dimensões, totalizando dez questões, o escore total varia de 07 a 49 pontos, sendo classificado em capacidade baixa, moderada, boa e ótima. Utilizou-se a versão do ICT traduzida e adaptada transculturalmente11 e validada para o português brasileiro. ${ }^{12-14}$

0 QFY tem por objetivo analisar a fadiga ocupacional mediante a interpretação subjetiva, realizada através da percepção de sentimentos e sensações específicas do cansaço, além dos indicadores comportamentais. ${ }^{15} 0$ mesmo contém seis dimensões com trinta subitens, seu escore total varia entre 30 e 150 pontos, sendo o valor máximo referente à maior fadiga e o escore mínimo correspondente à menor fadiga. ${ }^{16}$

\section{Análise dos dados}

Os dados numéricos foram apresentados sob a forma de média e desvio padrão ou mediana e amplitude. Os dados categóricos foram apresentados em percentuais. Os dados foram analisados por meio do pacote estatístico SPSS versão 19.0 (IBM Corp., Armonk, Estados Unidos). Aplicou-se o teste de Kolmogorov-Smirnov para verificar a normalidade da distribuição. Foi utilizado o teste de qui-quadrado para verificar a associação entre variáveis demográficas e problemas de sono com a fadiga e também foi verificada a associação da fadiga com o ICT. Quando a frequência esperada no teste foi inferior a cinco, foi utilizado o Exato de Fisher com extensão de Freeman-Halton. Em todas as análises foi considerado intervalo de confiança de 95\% (IC95\%) e nível de significância menor ou igual a $5 \%$ (p-valor $<0,05)$.

\section{Aspectos éticos}

A pesquisa foi avaliada e aprovada pelo Comitê de Ética em Pesquisa da UEPB, de acordo com o CAEE 0352.0.133. Os professores assinaram um Termo de Compromisso Livre e Esclarecido em 
respeito aos aspectos éticos relativos à pesquisa com sujeitos humanos, conforme a Resolução 466/12.

\section{RESULTADOS}

Dos 151 professores resultantes do cálculo amostral, 89 docentes compuseram a amostra. Pode-se destacar que a maioria era do sexo feminino (59,6\%), predominantemente, na faixa etária de 40 a 49 anos (40,4\%), casados $(64 \%)$ e possuíam filhos $(77,5 \%)$. A média de idade da população estudada foi de 47,74 \pm 8,08 anos (mediana de 47 anos, mínimo de 34 e máximo de 65).

Quanto ao nível educacional, observou-se predomínio de professores doutores (61,8\%). Estes docentes estavam lotados no Departamento de Fisioterapia (29,2\%), Farmácia (25,8\%), Odontologia (15,7\%), Educação Física (12,4\%), Biologia (9\%) e Psicologia (7,9\%).

Com referência aos anos de profissão, observou-se média de 19,52 $\pm 9,73$ anos (mediana de 20, mínimo de 2 e máximo de 43 anos). Foi evidenciado que a maioria dos docentes trabalhavam mais de um turno $(96,6 \%)$, com carga de trabalho semanal de $40 \mathrm{~h}$ (94,4\%). Destes, 18 (20,5\%) relataram desempenhar atividade laboral extra. Além disso, a renda mensal média da amostra foi de 11,18 $\pm 3,17$ salários mínimos (mediana de 10, mínimo de 3 e máximo de 20).

Considerando os hábitos de vida, a maior parte da amostra $(95,5 \%)$ relatou não fumar, apenas 38,2\% consomem bebida alcoólica e $33,7 \%$ possuem problemas para dormir. Dos professores entrevistados, 37,1\% dormem seis horas por noite, com uma média de $6,48 \pm 1,129$ (mediana de 6 , mínimo de 3 e máximo de 8 ) horas de sono.

Considerando a capacidade para o trabalho autorrelatada verificada através do escore total do ICT, foi de $40,19 \pm 4,446$ pontos (mediana de 41, mínimo de 28 a 49), sendo 49 a pontuação máxima. Ao analisar a distribuição da amostra foi visto que a maioria $(70,8 \%)$ considerava possuir uma boa capacidade para o trabalho (Tabela 1). Considerando a autoavaliação da capacidade para o trabalho, ao responder o questionamento "quantos pontos você daria para sua capacidade para o trabalho atual", foi visto

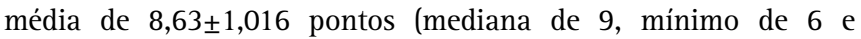
máximo de 10).

\section{Tabela 1.}

Distribuição dos docentes de acordo com o Índice de Capacidade para o Trabalho (ICT).

$\begin{array}{lcc}\text { ICT Classificação } & \text { N } & \% \\ \text { Moderada capacidade } & 21 & 23,6 \\ \text { Boa capacidade } & 63 & 70,8 \\ \text { Ótima capacidade } & 5 & 5,6 \\ \text { Total } & 89 & 100,00\end{array}$

Tabela 2.

Caracterização dos docentes de acordo com a fadiga, por seção, considerando média, desvio padrão e valor mínimo-máximo.

$\begin{array}{lrcc}\text { Fadiga } & \text { Média } & \text { Desvio Padrão } & \text { Mínimo-Máximo } \\ \text { Seção I* } & 21,72 & 6,047 & 11-37 \\ \text { Seção II** } & 21,93 & 5,555 & 10-39 \\ \text { Seção III** } & 19,82 & 5,297 & 10-35 \\ \text { Escore Total } & 63,47 & 15,063 & 34-105 \\ \text { * Sonolência e falta de disposição para o trabalho; ** Dificuldades de concentração e atenção; } \\ \text { **** Projeções da fadiga sobre o corpo. }\end{array}$

Em relação ao nível de fadiga laboral, foi observado por meio do QFY, que a média do escore total da fadiga dos docentes foi de $63,47 \pm 15,063$ pontos. Entretanto, a seção que obteve maior média de 21,93 $\pm 5,55$ pontos, está relacionado com a fadiga que envolve a dificuldade de concentração e atenção (Tabela 2). Ressalta-se o fato de que cada seção apresenta o valor máximo de 50 pontos.

Em relação ao nível de fadiga laboral, foi observado por meio do QFY, através da divisão por percentil que a maioria $(49,4 \%)$ dos docentes apresentou fadiga moderada.

Considerando o ICT e o QFY, observou-se correlação moderada e negativa do valor total da escala $(r=-0,48 ; p<0,0001)$. Quando considerados os tipos de fadiga, observou-se relação semelhante com o ICT (Tabela 3). Esses resultados indicam que quanto maior os niveis de fadiga, pior desempenho quanto à capacidade para o trabalho.

A fadiga física está relacionada com a ocorrência de sonolência e falta de disposição para o trabalho, sendo esta observada na Seção I do QFY. A fadiga mental corresponde à seção II, expressa através de dificuldade de concentração e atenção, sendo esta a que apresentou maior correlação moderada e negativa quando comparada aos demais niveis de fadiga. A fadiga geral refere-se à projeção da fadiga sobre o corpo, vista na Seção III do QFY.

Tabela 3.

Correlação do Índice de Capacidade para o Trabalho com o Questionário de Fadiga de Yoshitake.

\begin{tabular}{|c|c|c|c|}
\hline & ICT & $r$ Pearson & $p$-valor \\
\hline QFY total & & $-0,48$ & $<0,0001$ \\
\hline \multicolumn{4}{|l|}{ Fadiga } \\
\hline Fisica & & $-0,39$ & $<0,001$ \\
\hline Mental & & $-0,46$ & $<0,0001$ \\
\hline Geral & & $-0,43$ & $<0,0001$ \\
\hline
\end{tabular}

$\mathrm{R}$ Pearson $=$ resultado do teste de correlação de Pearson; $p$-valor= nível de significância.

Tabela 4.

Associação entre as variáveis sociodemográficas e Questionário de Fadiga de Yoshitake.

\begin{tabular}{|c|c|c|c|c|c|}
\hline Variáveis & $\begin{array}{l}\text { Frequência } \\
\text { \% }\end{array}$ & $\begin{array}{l}\text { Questionár } \\
\text { Baixa (\%) }\end{array}$ & $\begin{array}{l}\text { io de Fadiga } \\
\text { Moderada }(\%)\end{array}$ & Alta (\%) & $p^{*}$ \\
\hline Sexo & & & & & 0,004 \\
\hline Feminino & 59,6 & 13,2 & 56,6 & 30,2 & \\
\hline Masculino & 40,4 & 44,4 & 38,9 & 16,7 & \\
\hline Grupo Etário & & & & & 0,351 \\
\hline 30 a 39 anos & 16,9 & 26,7 & 60,0 & 13,3 & \\
\hline 40 a 49 anos & 40,4 & 16,7 & 47,2 & 36,1 & \\
\hline 50 a 59 anos & 36,0 & 31,3 & 50,0 & 18,8 & \\
\hline 60 ou mais & 6,7 & 50,0 & 33,3 & 16,7 & \\
\hline Estado Civil & & & & & 0,399 \\
\hline Com cônjuge & 64,0 & 29,8 & 49,1 & 21,1 & \\
\hline Sem cônjuge & 36,0 & 18,8 & 50,0 & 31,3 & \\
\hline Nivel educacional & & & & & 0,454 \\
\hline Graduado/especialista & 14,6 & 46,2 & 30,8 & 23,1 & \\
\hline Mestre & 16,9 & 26,7 & 53,3 & 20,0 & \\
\hline Doutor/Pós-doutor & 68,5 & 21,3 & 52,5 & 26,2 & \\
\hline Problemas para dormir & & & & & 0,000 \\
\hline Sim & 33,7 & 10 & 36,7 & 53,3 & \\
\hline Não & 66,3 & 33,9 & 55,9 & 10,2 & \\
\hline Fuma & & & & & 0,560 \\
\hline Sim & 4,5 & 50,0 & 25,0 & 25,0 & \\
\hline Não & 95,5 & 24,7 & 50,6 & 24,7 & \\
\hline Bebe & & & & & 0,453 \\
\hline Sim & 38,2 & 26,5 & 55,9 & 17,6 & \\
\hline Não & 61,8 & 25,5 & 45,5 & 29,1 & \\
\hline
\end{tabular}


Considerando as variáveis sociodemográficas, foi visto que as variáveis sexo $(\mathrm{p}<0,004)$ e ocorrência de problemas para dormir $(\mathrm{p}<0,000)$ demonstraram associação estatisticamente significativa $(p<0,05)$ com o escore do QFY.

\section{DISCUSSÃO}

A capacidade para trabalho é definida pela interação entre aspectos ocupacionais, pessoais e fatores relativos ao estado de saúde. ${ }^{17}$ Desse modo, espera-se que altos níveis de fadiga física e mental estejam associados à elevada carga de trabalho e elevada demanda emocional. ${ }^{18}$ Entretanto, observa-se que os indivíduos que compõem a amostra apresentaram uma boa capacidade para o trabalho e um moderado nível referido de fadiga, sobretudo relacionado à fadiga mental.

Os docentes que compõem a amostra estão inseridos em uma faixa etária caracterizada pela elevada produtividade e criatividade $(40,4 \%$ encontram-se entre 40 a 49 anos). Sendo assim, estão em um período considerado bastante produtivo, no qual evidencia-se o desenvolvimento das potencialidades relativas ao trabalho. ${ }^{19}$

Foi encontrada associação do sexo com a ocorrência dos maiores índices de fadiga. Isto é evidenciado pela predominância do sexo feminino no ambiente acadêmico, caracterizando assim, a tendência de feminização da docência. ${ }^{20}$ Além disso, deve-se considerar o existente predomínio histórico das mulheres nas áreas biológicas e da saúde devido à associação com o ato de cuidar das pessoas. $^{21}$

Diferentemente de estudos que relacionam a capacidade para o trabalho em professores do da educação básica ${ }^{22}$, observa-se que no ensino superior há predomínio de professores com doutorado, isto ocorre devido ao fato de que a qualificação acadêmica por vezes está associada com a ascensão profissional, neste caso, considerando a carreira universitária. ${ }^{23}$

No estudo de Marqueze et $\mathrm{al}^{24}$ realizado com 154 docentes universitários, foi observado que a maioria (87\%) dos docentes apresentou boa ou ótima capacidade para o trabalho. Entretanto, os docentes da área de saúde apresentaram uma capacidade para o trabalho pior, quando comparada com as demais áreas, para estes autores, essa relação poderia ser justificada através da atuação paralela dos profissionais em serviços de saúde. Tal fato pode não ter sido significante considerando a amostra do referido estudo, uma vez que apenas 20,5\% dos docentes entrevistados desempenhavam atividade laboral extra.

$\mathrm{Na}$ amostra estudada, foi encontrada uma associação entre existência de problemas para dormir com a ocorrência de fadiga ocupacional. Entretanto, de acordo com Fischer et $\mathrm{al}^{25}$ a ocorrência de problemas no sono também está do nível de fadiga estatisticamente associadas ao ICT, além da presença de obesidade. Por outro lado, o estilo de vida não apresentou associação significante.

Em um estudo realizado com trabalhadores de enfermagem foi observado que grande parte da amostra apresentou inadequada capacidade para o trabalho (40,8\%). Além disso, cerca de 25,7\% dos trabalhadores apresentaram altos niveis de fadiga. Portanto, foi possivel verificar que a fadiga por sua vez pode acarretar redução na motivação, além de gerar repercussões na capacidade para o trabalho. ${ }^{26}$

Diferentemente do atual estudo, uma pesquisa realizada com trabalhadores da enfermagem observou a não existência de correlação estatisticamente significativa entre o sono e a fadiga referida. Por outro lado, foi observada associação relevante entre jornada semanal de trabalho e fadiga. ${ }^{27}$

Estudos evidenciam a maioria dos docentes trabalham por mais de um turno, gerando uma intensificação do trabalho de docentes universitários $^{28}$, e isto, pode repercutir no âmbito psicológico, devido ao desgaste e fadiga., ${ }^{2,3}$ Além dos turnos de trabalho, a realização de atividade laboral extra está relacionada com o desgaste físico e mental. ${ }^{1}$ Sendo a maioria do trabalho extra relacionado com atendimento em hospitais, clínicas e consultórios. ${ }^{4}$

Considerando que a capacidade para o trabalho consiste em um processo dinâmico e que sofre influência de diversos fatores, foi verificado que a existência de altos níveis de fadiga está relacionada com a baixa capacidade para o trabalho.

Diante disso, dentro da perspectiva de saúde do trabalhador, sabe-se que os docentes são expostos a longas jornadas de trabalho, atividade laboral extra, sobrecarga de trabalho, entre outros fatores que resultam em desgaste físico e mental. Além disso, um agravante ao aspecto da saúde desses professores é que a atividade laboral extra está na maioria das vezes relacionado com o atendimento em hospitais, clínicas e consultórios. Entretanto, observa-se que existe um período etário no qual os docentes encontram-se na chamada intensificação do trabalho acadêmico, favorecendo os niveis da capacidade para o trabalho, entretanto, gerando repercussões quanto à ocorrência da fadiga ocupacional.

Estudo realizado na Universidade Estadual da Paraíba (UEPB), Campina Grande, PB, Brasil.

Financiamento próprio.

Não há conflitos de interesse. 


\section{REFERENCIAS}

1. Rezer CN, Donat MF, Ferretti F, Braghini CC. Condições de trabalho e saúde de professores do ensino superior no oeste catarinense. FisiSenectus. 2013;1(spec):85-95.

2. Fontana RT, Pinheiro DA. Condições de saúde auto-referidas de professores de uma universidade regional. Rev Gaúcha Enferm. 2010;31(2):270-276.

3. Koetz L, Rempel C, Périco E. Qualidade de vida de professores de Instituições de Ensino Superior Comunitárias do Rio Grande do Sul. Cien Saude Colet. 2013;18(4):1019-1028.

4. Cruz RM, Lemos JC, Welter MM, Guisso L. Saúde docente, condições e carga de trabalho. REID. 2010;4:147-160.

5. Hilleshein EF, Souza LM de, Lautert L, Paz AA, Catalan VM, Teixeira MG, et al. Capacidade para o trabalho de enfermeiros de um hospital universitário. Rev Gaúcha Enferm. 2011;32(3):509-515.

6. Servilha EAM, Arbach M de P. Queixas de saúde em professores universitários e sua relação com fatores de risco presentes na organização do trabalho. Distúrb Comun. 2011;23(2):181-191.

7. Mendonça VLG De, Coelho JAPDM, Júca MJ. Síndrome de Burnout em médicos docentes de uma instituição pública. Psicol Pesq [on line]. 2012 [cited 2014 May 13];6(2):90-100. Available from: http://pepsic.bvsalud.org/scielo.php?script=sci_ arttextCtpid=S1982-12472012000200002\&lng=pt\&nrm=i\&tIng=pt

8. Nery D, Toledo AM, Oliveira Júnior $S$, Taciro $C$, Carregaro R. Análise de parâmetros funcionais relacionados aos fatores de risco ocupacionais da atividade de enfermeiros de UTI. Fisioter Pesq. 2013;20(1):76-82.

9. Martinez MC, Latorre M do RD de 0, Fischer FM. Capacidade para o trabalho: revisão de literatura. Cien Saude Colet. 2010;15(Suppl.1):1553-1561.

10. Figlioulo DS da S, Lima PO de P, Laurentino GEC. Estresse ocupacional e fadiga em fisioterapeutas que exerciam função de docência em universidade da cidade de Recife - PE. Ter Man. 2011;9(41):22-28.

11. Tuomi K, IImarinen J, Katajarinne L. Índice de Capacidade para o Trabalho. São Carlos: EDUFSCAR; 2005.

12. Martinez MC, Latorre MRDO, Fischer FM. Validade e confiabilidade da versão brasileira do Índice de Capacidade para o Trabalho. Rev Saúde Pública. 2009;43(3):525-532.

13. Silva Junior SHA da, Vasconcelos AGG, Griep RH, Rotenberg L. Validade e confiabilidade do indice de capacidade para o trabalho (ICT) em trabalhadores de enfermagem. Cad Saúde Pública. 2011;27(6):1077-1087.

14. Silva Júnior SHA da, Vasconcelos AGG, Griep RH, Rotenberg L. Confiabilidade teste-reteste do İndice de Capacidade para o Trabalho ( ICT ) em trabalhadores de enfermagem. Rev Bras Epidemiol. 2013;16(1):202-209.
15. Yoshitake H. Three Characteristic Patterns of Subjective Fatigue Symptoms. Ergonomics [on line]. 1978 [cited $2017 \mathrm{Mar}$; 21(3):231-233. Available from: http://www.tandfonline.com/doi/abs/10.1080/00140137808931718

16. Metzner RJ, Fischer FM. Fadiga e capacidade para o trabalho em turnos fixos de doze horas. Rev Saúde Pública. 2001;35(6):548-553.

17. Seibt R, Spitzer S, Blank M, Scheuch K. Predictors of work ability in occupations with psychological stress. J Public Health (Bangkok). 2009;17(1):9-18.

18. Arvidsson I, Håkansson C, Karlson B, Björk J, Persson R. Burnout among Swedish school teachers - a cross-sectional analysis. BMC Public Health [on line]. 2016 [cited $2017 \mathrm{Mar}$;:16(1):823. Available from: http://bmcpublichealth.biomedcentral.com/articles/10.1186/s12889-016-3498-7

19. Contaifer TRC, Bachion MM, Yoshida T, Souza JT de. Estresse Em Professores Universitários Da Área De Saúde. Rev Gaúcha Enferm. 2003;24(2):215-225.

20. Carneiro RM. Sindrome De Burnout : Um Desafio Para 0 Trabalho Do Docente [dissertação]. Anápolis (G0): Centro Universitário de Anápolis; 2010.

21. Souza MDL De, Sartor WDB, Padilha MICDS, Prado ML Do. 0 Cuidado em Enfermagem: uma aproximação teórica. Texto Context-Enferm [on line]. 2005 [cited $2017 \mathrm{Mar}$; 14(2):266-270. Available from: http://www.scielo.br/pdf/tce/ v14n2/a15v14n2.pdf

22. Pereira ÉF, Teixeira CS, Pelegrini A, Meyer C, Andrade RD, Lopes A da S. Estresse relacionado ao trabalho em professores de educação básica. Cienc Trab. 2014;16(51):206-210.

23. Lopes MJM, Leal SMC. A feminização persistente na qualificação profissional da enfermagem brasileira. Cad Pagu. 2005;24(1):105-125.

24. Marqueze EC, Moreno CR de C. Satisfação no trabalho e capacidade para o trabalho entre docentes universitários. Psicol Estud. 2009;14(1):75-82.

25. Fischer F, Borges N, Rotenberg L, Latorre $M$, Soares NS, Rosa $P$, et al. A (in) capacidade para o trabalho em trabalhadores de enfermagem. Rev Bras Med Trab (Belo Horiz). 2005;3(2):97-103.

26. Vasconcelos SP, Fischer FM, Reis AOA, Moreno $C R$ de $C$. Fatores associados à capacidade para o trabalho e percepção de fadiga em trabalhadores de enfermagem da Amazônia Ocidental. Rev Bras Epidemiol. 2011;14(4):688-697.

27. Rosa PLFS, Fischer FM, Borges FN da S, Soares N da S, Rotenberg L, Landsbergis P. Percepção da duração do sono e da fadiga entre trabalhadores de Enfermagem. R Enferm UERJ. 2007;15(1):100-106.

28. Seabra MMA, Silva e Dutra FCM. Intensificação do Trabalho e Percepção da Saúde em Docentes de uma Universidade Pública Brasileira. Cienc Trab. 2015;17(54):212-218. 